\title{
Effect of Fluid-Structure Interaction on Noise Generation in MAV with Fixed and Flapping Membrane Wing
}

\author{
S. Syam Narayanan ${ }^{\dagger}$ and R. Asad Ahmed \\ KCG College of Technology, Chennai, Tamil Nadu, 600097, India \\ †Corresponding Author Email: syam.aero@kcgcollege.com
}

(Received April 5, 2021; accepted June 22, 2021)

\begin{abstract}
An experimental study on the effect of fluid-structure interaction on noise generation in Micro Air Vehicle (MAV) with fixed and flapping membrane Tipula sp. wing is investigated. The acoustic performance of the fixed and flapping wing which made up of certain characteristic thin materials such as Low-density Polyethylene Terephthalate (PET), Thin Aluminium sheets (Al), and Non-woven fabrics (NWF) is analysed. An acoustic study is conducted to estimate the acoustic characteristic parameters of the insect mimic- membrane wing for various flapping conditions with various flapping frequency. In this research, the membrane wing with $15 \mathrm{~cm}$ of the total span is tested on both fixed and flapping MAV at different flexibility conditions and velocity conditions. The study of flapping MAV enables the study of the characteristic effects of sound emitted during the flapping motion of a wing. With the analysed results, the performance of wings is identified and compared with the sound pressure level. After analysing different materials, it is found that NWF produces $20 \%$ less noise than the other two more materials. Since the stiffness to strength ratio of metal is high, the formation of vortices is less compared to other membranes. For all fixed membrane wings at low Strouhal numbers, the formation of vortices is very low, and when the Strouhal number increases, the vortices became dense and results in the reduction of Sound pressure level.
\end{abstract}

Keywords: Fluid-structure interaction; Aerodynamics loading; Flapping; Unsteady flow; Vortex flow.

\section{NOMENCLATURE}

$\begin{array}{ll}A l & \text { aluminum sheet } \\ c_{f} & \text { unstiffened chord } \\ c_{s} & \text { stiffened chord } \\ c^{\prime} & \text { extended imaginary chord } \\ c^{*} & \text { mean aerodynamic chord } \\ f & \text { linear flapping frequency } \\ k^{*} & \text { flexibility constant } \\ \text { LAeq } & \text { level A-weighted equivalent }\end{array}$

\section{INTRODUCTION}

Micro air vehicles are the tiny form of the unmanned air vehicle. These types of micro-air vehicles are suitable for military surveillance and aerial photography, because of its small size (limited to $15 \mathrm{~cm}$ span), weight ( $\max$ of $20 \mathrm{gm}$ ), low noise and the ability to escape from the radar. Due to its small and flexible wing, the aerodynamic characteristics of the MAV will change at different fluid conditions. These fluid interactions will deform its wing

\author{
NWF Non-Woven Fabric \\ PETS Polyethylene Terephthalate Sheet \\ $R e \quad$ Reynold's Number \\ $R F \quad$ Radio Frequency \\ $S P L \quad$ Sound Pressure Level \\ $\Gamma \quad$ dihedral angle \\ $\delta \quad$ plunging angle \\ $\theta$ angular location around the vehicle
}

structure and it affects the aerodynamic performances and vice versa. Nowadays, most unmanned Arial vehicles are moving to a smaller size because it has many advantages. These types of air vehicles are normally operated at a low Reynolds number and the materials used for the wing structure are thin and lightweight. Hence the effect of fluidstructure interactions plays an important role in the aerodynamics performance. (Ifju et al. 2001; Narayanan et al. 2020; Lian 2003). The nature of rigid and membrane wings in MAV. In their 
research, three different types of wings with different stiffness factors are analyzed. It is observed that stiffened wing exhibits a higher lift coefficient than a flexible membrane wing. (Liebeck 1992; McIntosh et al. 2006) The membrane wing efficiency is effectively designed by Ifju et al. (2002). Narayanan et al. (2019), analysed six types of membrane wings similar to insects' wings and observed that, the Tipula wing is producing a higher lift coefficient compare to the other wings. DeLaurier (1993) was discovered that a specific mechanism was equipped for fluttering at higher frequencies than others. This sort of pitch movement differs between the mechanisms also; some can powerfully change pitch, while others have fixed pitch envelopes. (Miller and Peskin 2009) A portion of the data gained from machines structured by others was joined in later sections, which clarifies the plan procedure of the component for this proposal. Various small fixedwing UAVs are being used today for an assortment of utilizations. A streamlined design would straightforwardly affect the endurance. Then again, an air acoustic improvement may build attentiveness in military working conditions. Some journals introduced few methods for the plan of silent rotors for MAV, from aerodynamic expectation to air acoustic improvement (Bae and Moon (2008). Zhenbo et al. (2014) did an acoustic investigation on a honey bee. This examination explores the honey bee sound during flapping conditions. They discovered that most of the noise is produced by the transverse and extraneous movements of the wing. The wing vortex interactions make minimum sound, results in a higher lift coefficient that permits the reduction of the rotational speed. This will result in the reduction of flapping frequency and the formation of vortices. Zhenbo et al. (2017) did the acoustic study of various wing structures for a twowinged fluttering wing miniaturized scale air vehicle. They watched changes in the geometry and structure of this wing for improving its efficiency but it didn't appear to unfavorably influence its acoustic reduction.

\section{EXPERIMENTAL ANALYSIS}

An acoustic study of membrane wing of $0.15 \mathrm{~m}$ of total span length is tested at different free stream conditions. Testing is conducted on fixed-wing and flapping MAV for different flexibility, velocity conditions, and flapping frequencies. This enables the study of characteristic effects of sound emitted during the flapping motion of a UAV. With the analysed results, the performance of wings was identified and compared with the sound pressure level. An Experimental study was made on an acoustic performance OF a Micro Aerial Vehicle with a Tipula sp. insect wing. Analysis was on the insect wing made up of certain characteristic thin materials such as Polyethylene terephthalate sheet (PET), Aluminium sheets (Al), Non-woven fabrics (NWF). (Fig. 1)

Throughout the investigation, the following limits or assumptions should be made while comparing fixed and flapping MAVs. The flapping mechanism is solely based on plunging rather than pitching, and the wings are flat plate with a dihedral angle of 100 . Furthermore, observing the accurate noise of flapping wings in a MAV, thereby investigating the wing with various materials (Table 1) with different stiffened conditions. Thereafter estimating how the changes in material properties influence the effect of sound generation in flapping. Then, consolidating the materials based on the noise generation in the flapping motion. Then, to estimate the wing material and $k^{*}$ (Eq.1) with the least sound produced during the flight. Where $k^{*}$ is defined as the ratio between the stiffened and unstiffened chord of the wing structure.

$k^{*}=C_{s} / C_{f}$

Therefore, making it reliable in spying and surveillance-related application for improving its capabilities by reducing the characteristic effect of noise emitted during the flapping motion of a MAV.

Table 1 Properties of the materials used for the fabrication of membrane wing

\begin{tabular}{|l|c|c|c|}
\hline \multicolumn{1}{|c|}{ Properties } & Al & NWF & PET \\
\hline Specific gravity & 1.32 & 0.98 & 2.7 \\
\hline Thickness & 0.04 & 20 & 0.1 \\
\hline Areal density & 124.45 & 98.65 & 102.45 \\
\hline Tensile strength & $80-100$ & $35-60$ & $50-150$ \\
\hline E modulus & $2000-2500$ & 1750 & 70000 \\
\hline
\end{tabular}

Fabricated models are tested under the flapping condition on sound restriction chamber and fixedwing with the different stiffened condition using blower type wind tunnel

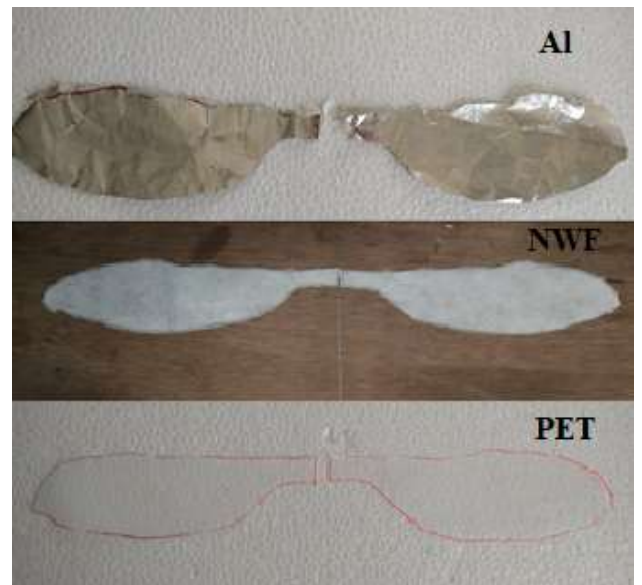

Fig. 1. Tipula sp. membrane wing made of $\mathrm{Al}$, NWF and PET.

External sound restrictor chamber works on a similar principle has an anechoic chamber which is primarily used to restrict the sound disturbance being heard inside the chamber. This chamber is enclosed by external shielding, which provides isolation from the 
outside environment. It is used to conduct experiments. This is constructed with echo suppression features and with effective isolation from the acoustic or RF noises that are present in the external environment. This combination allows exclusively to hear direct sounds (no reverberant sounds), simulating being inside an infinitely large room. This ensures the integrity of the experiment carried out inside as it is not influenced by external or internal reflected noise.

A microphone is a transducer that converts acoustical waves into electrical signals. It is used to measure sound emitted during the flapping and fixed-wing experiment. In this experimentation, an ear pod microphone is used and a condenser microphone is used to measure the acoustic waves. A condenser microphone operates on a capacitive design. It incorporates a stretched metal diaphragm that forms one plate of a capacitor. A metal disc placed close to the diaphragm acts as a backplate. When a sound field excites the diaphragm, the capacitance between the two plates varies by the variation in the sound pressure.

A sound level meter is used for acoustic (sound that travels through the air) measurements. It is commonly a hand-held instrument with a microphone. The best type of microphone for sound level meters is the condenser microphone, which combines precision with stability and reliability. The diaphragm of the microphone responds to changes in air pressure caused by sound waves. That is why the instrument is sometimes referred to as a Sound Pressure Level (SPL) Meter.

Z-Weighting represents the actual sound produced, A-Weighting, with less low and higher frequencies and a slight boost in the mid-range, represents what humans are capable of hearing, C-Weighting, more sensitive to the lower frequencies, represents what humans hear when the sound is loud.

Sound Analyzer is an application developed in an android platform as a Sound Level Meter (SLM) and a Real-time Audio Analyzer (RTA). Environmental noise measured in decibel (dB) can be monitored in real-time. The microphone sensitivity is adjustable using the calibration menu. The mounting system used to mount and hold the model in an erect position to support the model vertically to the ground axis and to ensure the model not get interrupted during the experimentation process (Fig. 2).

The fixed-wing model is placed in front of the blower so that, air flows against it. The steel stand is fixed to hold the fixed-wing model in its position to ensure that it doesn't move till testing completes. The microphone is placed in a different position to measure the sound emitted during testing (Fig. 3). The microphone is connected to an android Sound Analyser Application. The Sound Analyzer App will use an android device as a Sound Level Meter (SLM) and a Real-time Audio Analyser (RTA). Environmental noise measured in decibel $(\mathrm{dB})$ can be monitored in real-time. The microphone sensitivity is adjustable using the calibration menu.

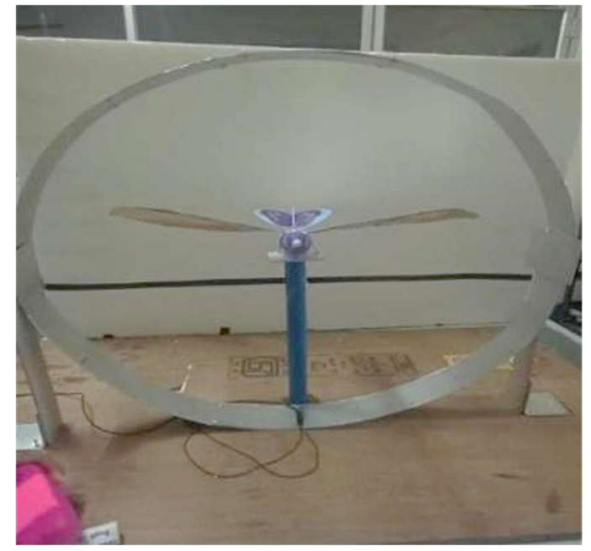

Fig. 2. Mounting stand and ring for sound measurement.

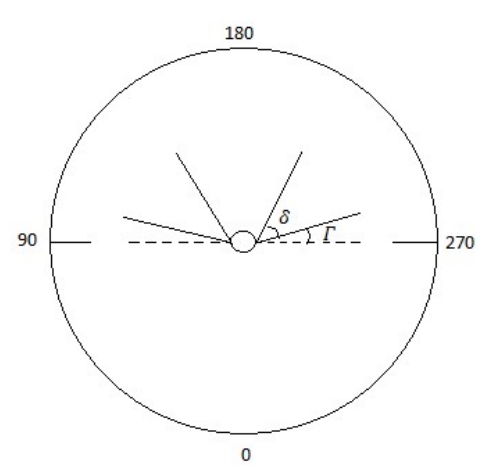

Fig. 3. Initial position of the Flapping MAV within the experimental setup.

The initial volume of the motor voice is calibrated before the initial operation of the experiment. Thus, the software application enables the calculation of the Sound Pressure Level (SPL) with maximum accuracy and 10-15 readings per second of the experimentation. For a fixed MAV sound pressure level has been measured at different extended imaginary chord (c') (Fig. 4). An extended imaginary chord is defined as the position of the microphone or observation point beyond the wingtip at the downstream of ai (Eq. 2). It is the function of the mean aerodynamic chord of the wing.

$c^{\prime}=c^{*}+\frac{1}{x} c^{*}$

where $\mathrm{x}=2,3,4 \ldots$ and $\mathrm{c}^{*}$ is the mean aerodynamic chord of the wing. In this study different SPL is measured at different c' of $0.25,0.5$, and $0.75 \mathrm{~mm}$. The flapping wing is mounted on the mount hold stand and the microphone is placed in the required angular position along the circumferential axis from 0 to $2 \pi$ angle with the variation of 30 degree in between every angular position. The position is adjusted after completing a series of the reading at the position. The flapping frequency is altered for every experimentation through the remote controller to change the rpm for the motor. The entire experimental setup is placed inside an External sound restrictor chamber which is primarily used to restrict the sound disturbance being heard inside the 
chamber. The initial atmospheric noise is calibrated before the initial operation of the experiment and set to nullify.

Free stream direction

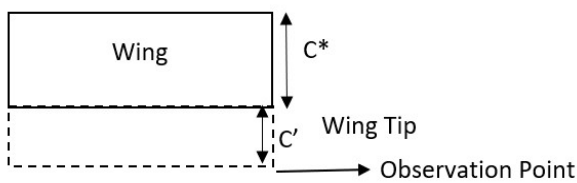

Fig. 4. Mounting stand and ring for sound measurement.

The values of the frequencies $\mathrm{A}, \mathrm{C}$, and $\mathrm{Z}$ (flat) frequency weightings, Fast and Slow time weightings, Equivalent A-weighted continuous sound level (LAeq), A-weighted Sound Exposure Level (SEL) $1 / 3$ octave band spectra: Centre frequencies $25 \mathrm{~Hz}$ to $16 \mathrm{kHz}, 1 / 1$ octave band spectra: Centre frequencies $31.5 \mathrm{~Hz}$ to $8 \mathrm{kHz}, \mathrm{A}, \mathrm{C}$, and $\mathrm{Z}$ (flat) frequency weightings, Fast, Slow and Impulse time weightings, Equivalent continuous sound level (LAeq) is measured through software which is coupled with a laptop to extract the data's through server sources and then the collected data are tabulated in the excel sheets for cumulative access of the data. Then the Equivalent A-weighted continuous sound level (LAeq) is chosen to compare with the calculated value of the other specifications of the wings designed for experimentation.

\section{RESULTS AND DISCUSSION}

Testing has been done on fixed-wing MAV for different flexibility and velocity conditions and as flapping wing MAV for hovering conditions at different flapping frequencies. This helped to study the characteristic effects of sound emitted during the flapping motion of a UAV. With the analyzed results, the performance of wings is identified and compared with the sound pressure level. The materials used for the wing fabrication are varied to test for the acoustic characteristics for three different materials with extreme material characteristics. They are classified as a metallic group, fabric group and polymer group. Aluminum is a versatile metal with a major advantage of its lightweight and flexible nature. An additional merit of aluminum is being easily recyclable and less corrosion rate amongst the rest of the metals. Thus, due to its extreme versatility and strength, it is used for the first type of wing specimen utilized for experimentation.

Acoustic studies were made for flapping and fixedwing at different $\mathrm{k}^{*}$ values. In Figs. 5-7, It is observed that for a fixed membrane wing MAV for all three materials, when the Reynolds number increases, the Sound pressure level also increases, it indicates fluttering of membrane wing increase with Reynolds number. At a high Reynolds number, the rate of change of SPL is high for the wing with lower $\mathrm{k}^{*}$.

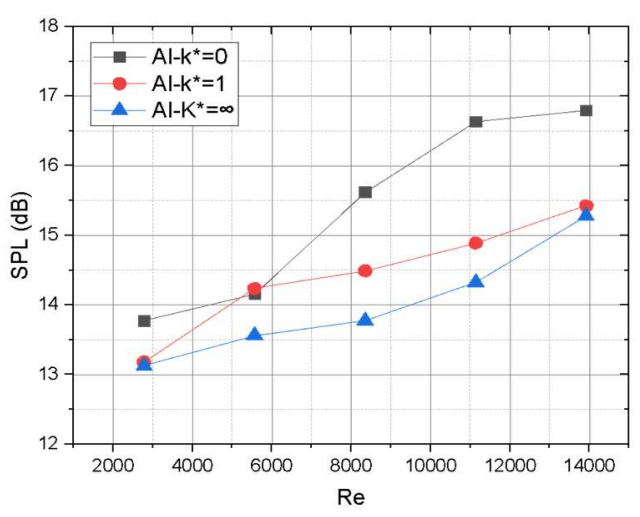

Fig. 5. Sound Pressure Level vs Reynolds Number (Re) in a Fixed Al membrane wing MAV with various $k^{*}$.

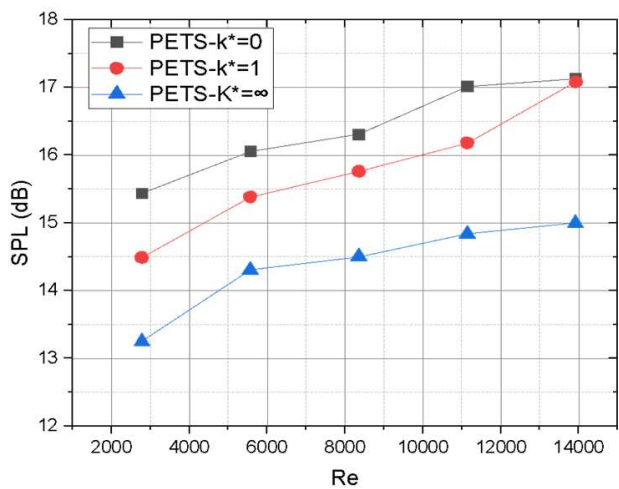

Fig. 6. Sound Pressure Level vs Reynolds Number (Re) in a Fixed PETS membrane wing MAV with various $k^{*}$.

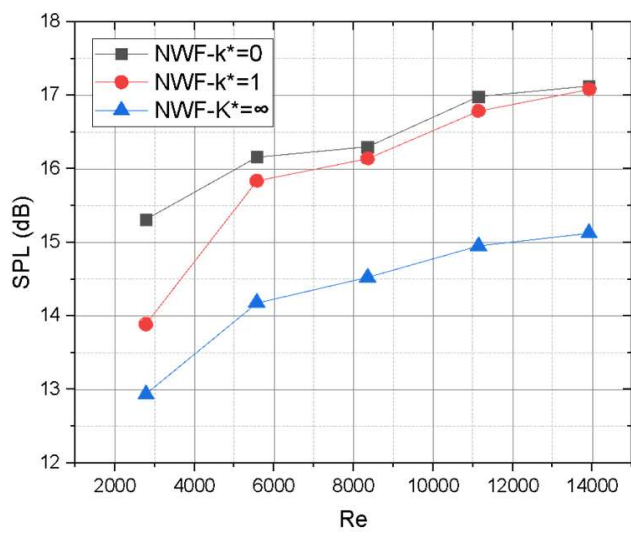

Fig. 7. Sound Pressure Level vs Reynolds Number (Re) in a Fixed NWF membrane wing MAV with various $k^{*}$.

On fixed MAV, the SPL of different $\mathrm{k}^{*}$ wings at different c' (extended imaginary chord defined in equation 3.8) has been measured. It is observed that the wing with the highest $\mathrm{k}^{*}$ is having the lowest sound due to its rigid nature when compared to the wing with the lowest $\mathrm{k}^{*}$ value (Figs. 8-15). The wing with low $\mathrm{k}^{*}$ produces 


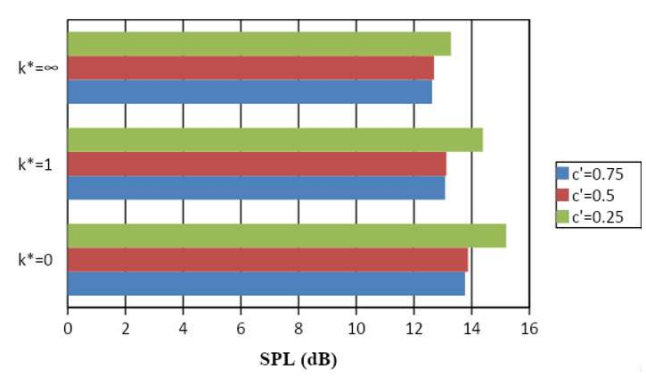

Fig. 8. Sound Pressure Level is measured on a fixed Al- membrane wing with various $k^{*}$ at different c' locations, flying at Reynolds Number of 2786 .

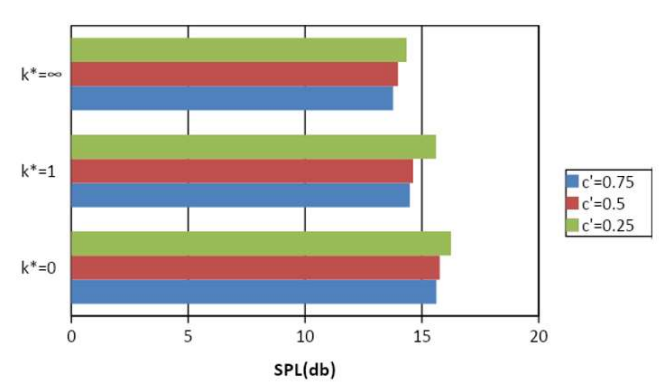

Fig. 9. Sound Pressure Level is measured on a fixed Al- membrane wing with various $k^{*}$ at different c' locations, flying at Reynolds Number of 8357 .

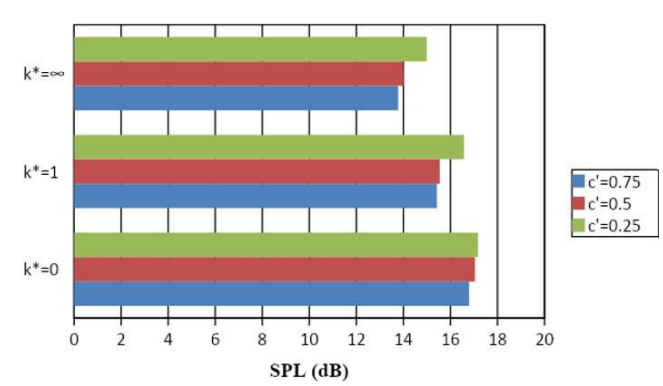

Fig. 10. Sound Pressure Level is measured on a fixed Al- membrane wing with various $k *$ at different c' locations, flying at Reynolds Number of 13928.

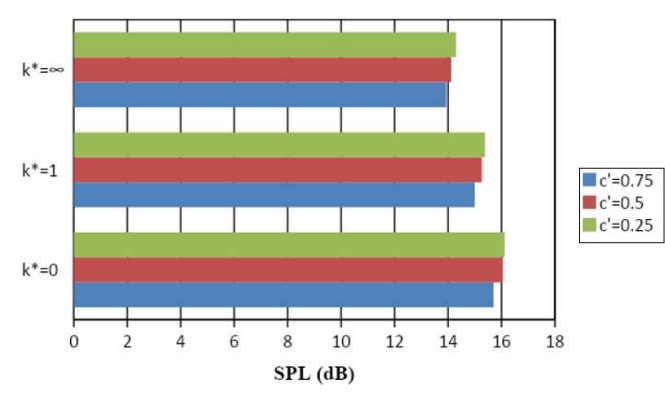

Fig. 11. Sound Pressure Level is measured on a fixed PETS membrane wing with various $k^{*}$ at different c' locations, flying at Reynolds Number of 5571 .

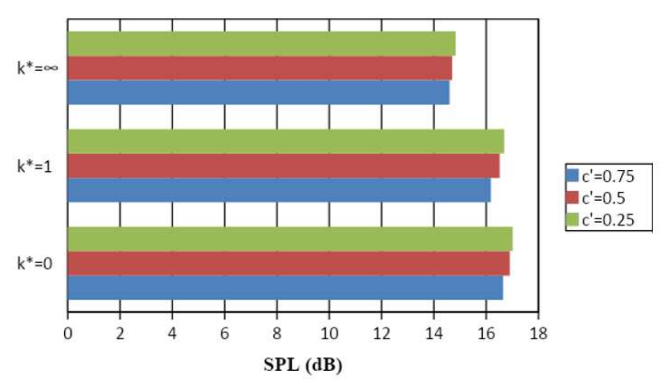

Fig. 12. Sound Pressure Level is measured on a fixed PETS membrane wing with various $k^{*}$ at different c' locations, flying at Reynolds Number of 11143 .

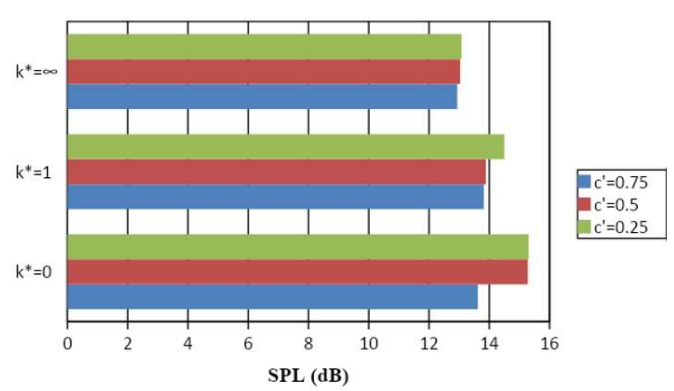

Fig. 13. Sound Pressure Level is measured on a fixed NWF membrane wing with various $k^{*}$ at different c' locations, flying at Reynolds Number of 2786 .

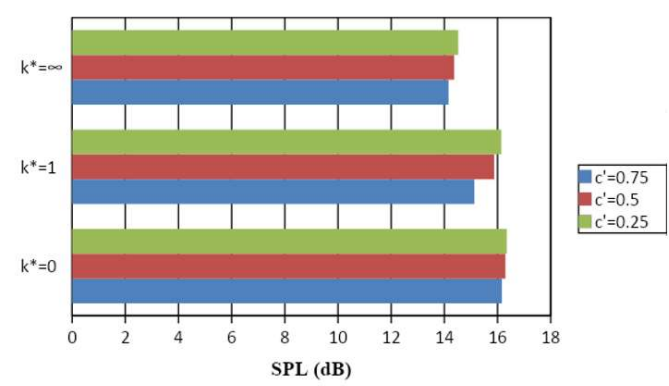

Fig. 14. Sound Pressure Level is measured on a fixed NWF membrane wing with various $k^{*}$ at different c' locations, flying at Reynolds Number of 8357.

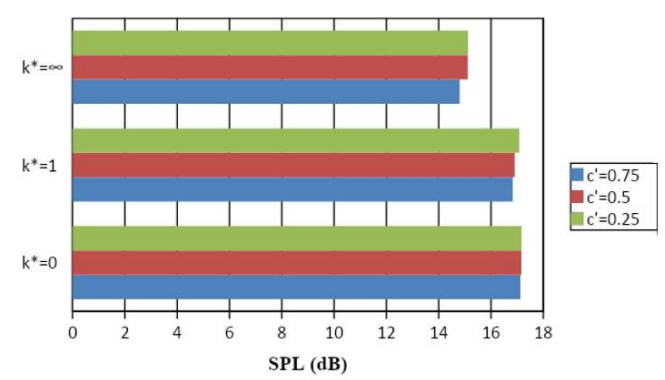

Fig. 15. Sound Pressure Level is measured on a fixed NWF membrane wing with various $k^{*}$ at different c' locations, flying at Reynolds Number of 13928. 
maximum SPL due to its fluttering nature. This can be seen in all three types of membrane wings of different materials. It is observed that when the c' increases, SPL decreases. This is the same for all Reynolds numbers and $\mathrm{k}^{*}$ value. Moreover, the wing made up of aluminum-like materials shows a very high variation. For high Reynold's number, the rate of change of SPL at different c' is reducing. This may be due to the high vortex shedding at the trailing edge of the wing. At high Reynold's number, the SPL value seems to be high, this sound is contributed by the noise generated by the free stream air.

From Figs. 16-21, the plot explains the location of the noise spectra along the flapping-wing MAV with the variable flapping frequencies for wing aluminum membrane wing. The variation of the sound profile along the circumferential axis is varying in a particular pattern of repetition. From the pattern of fluctuation, the peak is noted at 180 degrees, for all the flapping frequencies studied. On analyzing this pattern, the peak shoot might be due to the doubled effect of wing fluttering since the wing flaps upwards that is the only upstroke is possible in flapping. The maximum noise spectra are found at 180 degrees. The trough is estimated at an extreme opposite position that is 0 degrees, where the sound recorder is the least due to the absence of the downstroke of the flapping motion. In the case of combined stroke operation, the peak shoots would have been seen at either extreme of the circumferential axis.

Sound spectra were studied with flapping-wing MAV, at no wing and with type A of wing attached conditions. The noise spectra are measured at a location of 180 degrees and for various flapping frequencies. The noise level recorded without the wing condition is quite compared to with the wing. The sound recording is found as the result of combinations of mechanical meshing of the gears and its motor-generated sound. The plot depicts that the curve is almost linear at no wing condition.

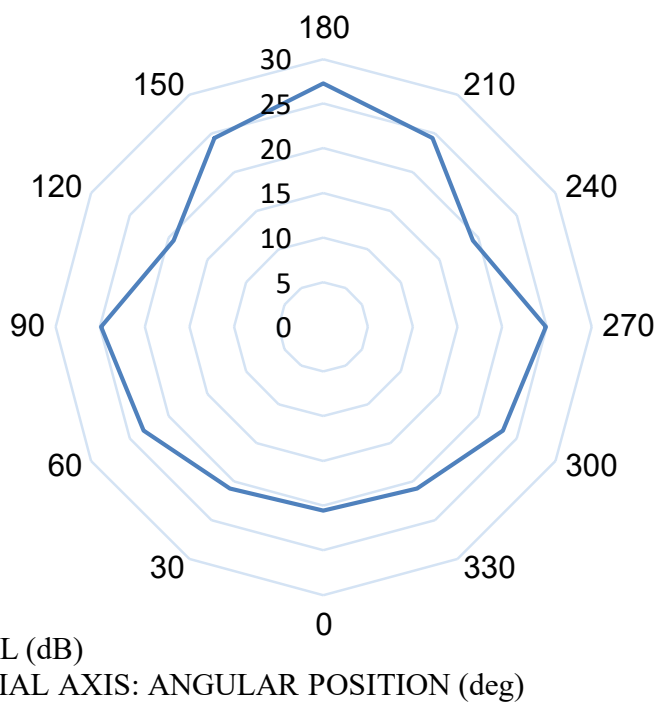

RADIAL AXIS:SPL (dB)

CIRCUMFERENTIAL AXIS: ANGULAR POSITION (deg)

Fig. 16. Distribution of SPL around the flapping PETS MAV wing of $f=2 \mathrm{~Hz}$.

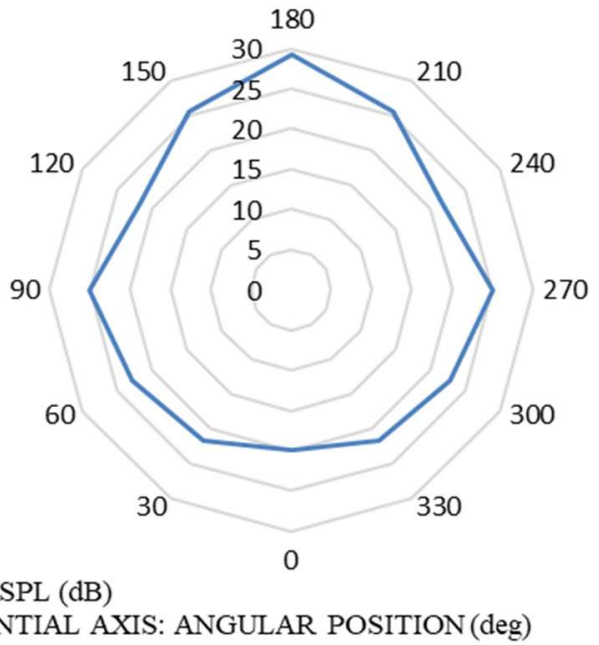

RADIAL AXIS:SPL (dB)

CIRCUMFERENTIAL AXIS: ANGULAR POSITION(deg)

Fig. 17. Distribution of SPL around the flapping PETS MAV wing of $f=4 \mathrm{~Hz}$. 
S. Syam Narayanan and R. Asad Ahmed/ JAFM, Vol. 14, No. 6, pp. 1817-1826, 2021.

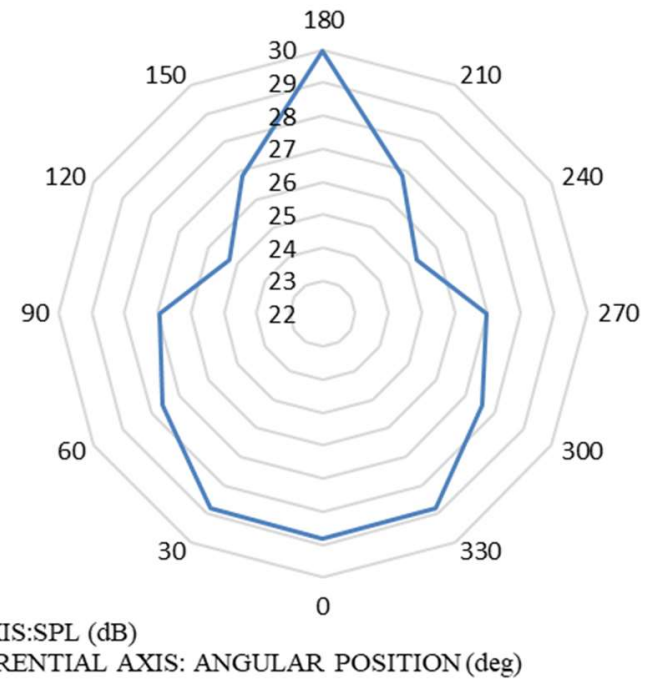

Fig. 18. Distribution of SPL around the flapping PETS MAV wing of $f=6 \mathrm{~Hz}$.

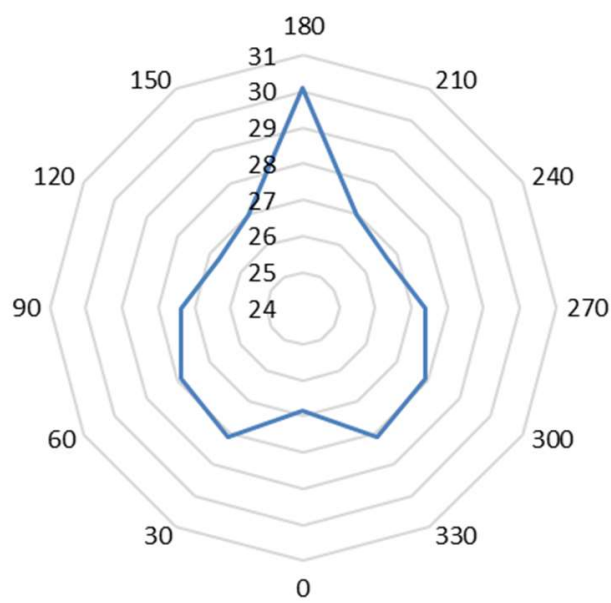

RADIAL AXIS:SPL (dB)

CIRCUMFERENTIAL AXIS: ANGULAR POSITION(deg)

Fig. 19. Distribution of SPL around the flapping PETS MAV wing of $f=8 \mathrm{~Hz}$.

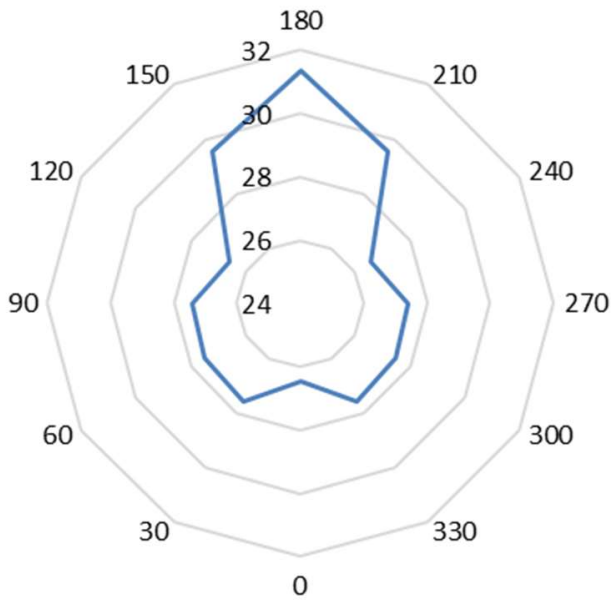

RADIAL AXIS:SPL (dB)

CIRCUMFERENTIAL AXIS: ANGULAR POSITION(deg)

Fig. 20. Distribution of SPL around the flapping PETS MAV wing of $f=10 H z$. 


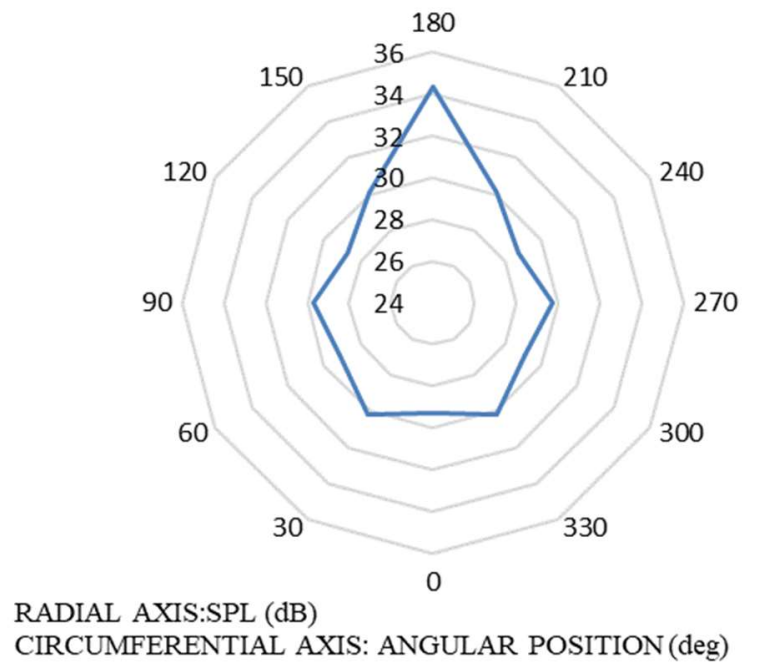

Fig. 21. Distribution of SPL around the flapping PETS MAV wing of $f=12 \mathrm{~Hz}$.

The plot (Figs. 22-24) elaborates that the variation of the noise observed in comparison is due to the fluidstructure interaction of the wing with airflow and additional vortices created during the flapping of the wing at varying frequencies levels. From the plot it is interpreted that the maximum slope of sound pressure level SPL is estimated across $2 \mathrm{~Hz}$ to $12 \mathrm{~Hz}$ of frequency of flapping because of the formation of the wing tip vortices is slower but attached to the wing surface, which is greatly influenced by the lower frequency of flapping.

The shift of the slope along the other frequencies is less significantly varying since a greater increase of the flapping frequencies has reduced their effect on the slope of the SPL during the flapping phase, In addition to the consequently faster detachment of generated vortices during flapping.

The sound Pressure level is proportional to the fluttering nature of the wing. At the lowest $\mathrm{k}^{*}$ value SPL is high because of its flexible nature. This chaotic nature of flexible wings creates boundary layer separations and the formation of local vortices. The highest $\mathrm{k}^{*}$ wing is producing low sound compare to the other wing conditions (Fig. 25). This means less local vortex formations in these wings. Rate of change of SPL with respect to flapping frequencies is more than the change due to Reynolds Number.

\section{Conclusion}

An acoustic study on membrane wing MAV, after analysing different materials, it is concluded that in the case of sound pressure level because of its stiffened nature, the wing with a high $\mathrm{k}^{*}$ value produces less sound. In the lowest $\mathrm{k}^{*}$ wing, the chaotic nature of the wing creates local vortices due to the boundary layer separation. The rate of change noise is directly proportional to flapping frequency. The study was made on the three kinds of materials: Aluminium, Polyethylene terephthalate, Non-woven material, among these Polyethylene terephthalate membrane wings produce more sound than the other two wings and Aluminum membrane wing produced less sound than the other two wings. NWF is reported to emit $20 \%$ less noise than the other two more materials. The difference in noise production between PETS and $\mathrm{Al}$ is 10 to $15 \%$. It can be seen that; the highest stiffened membrane wing is the best to develop flapping or fixed MAV.

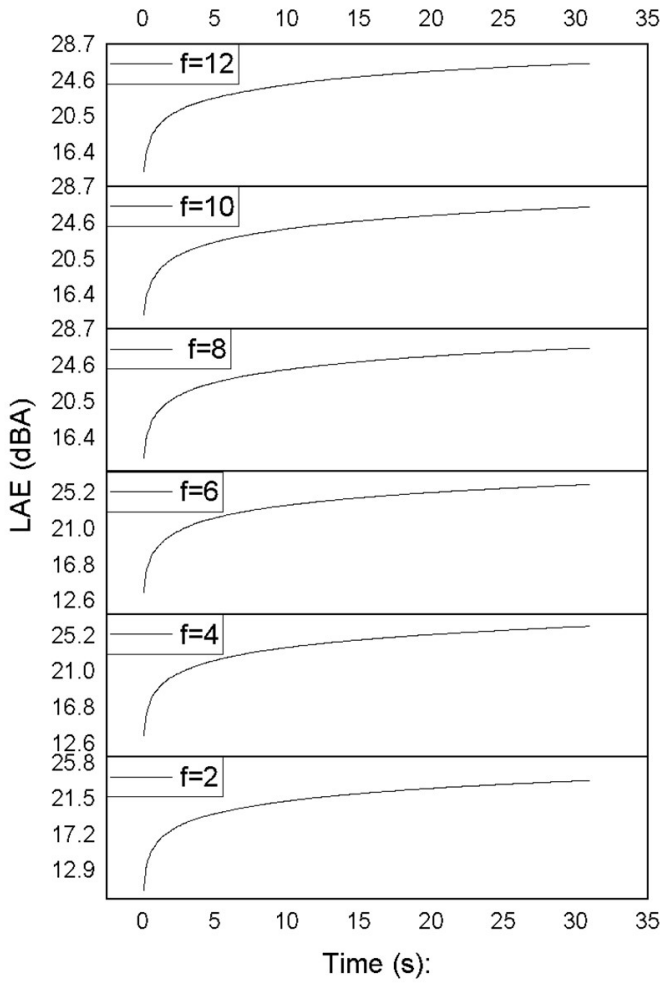

Fig. 22. Recorded noise level of Al-flapping wing MAV with respect to time at different flapping frequencies. 


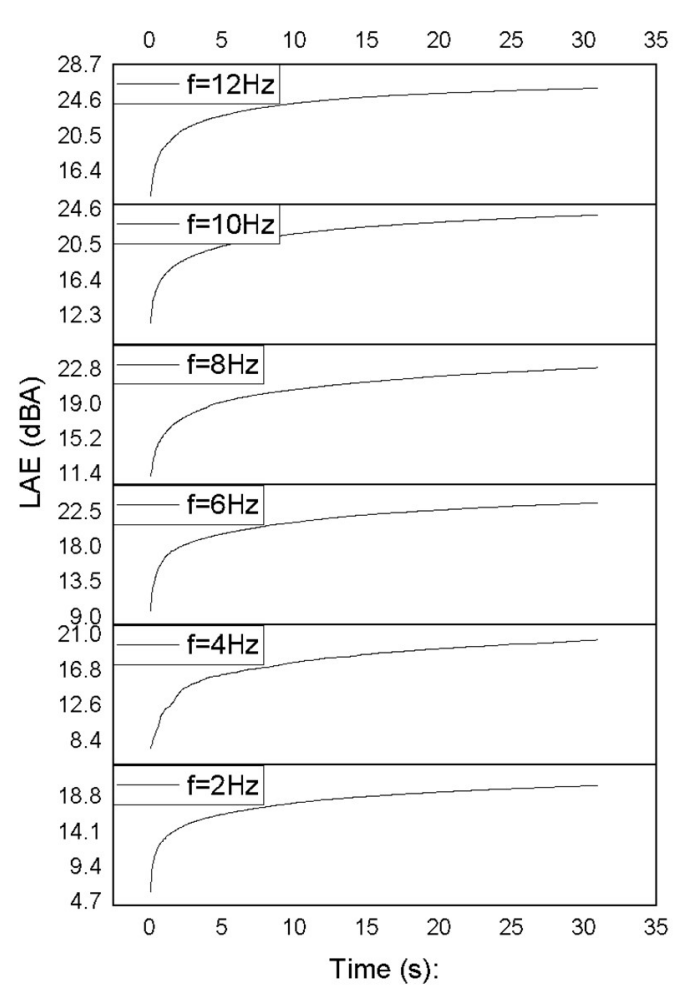

Fig. 23. Recorded noise level of NWF flappingwing MAV with respect to time at different flapping frequencies.

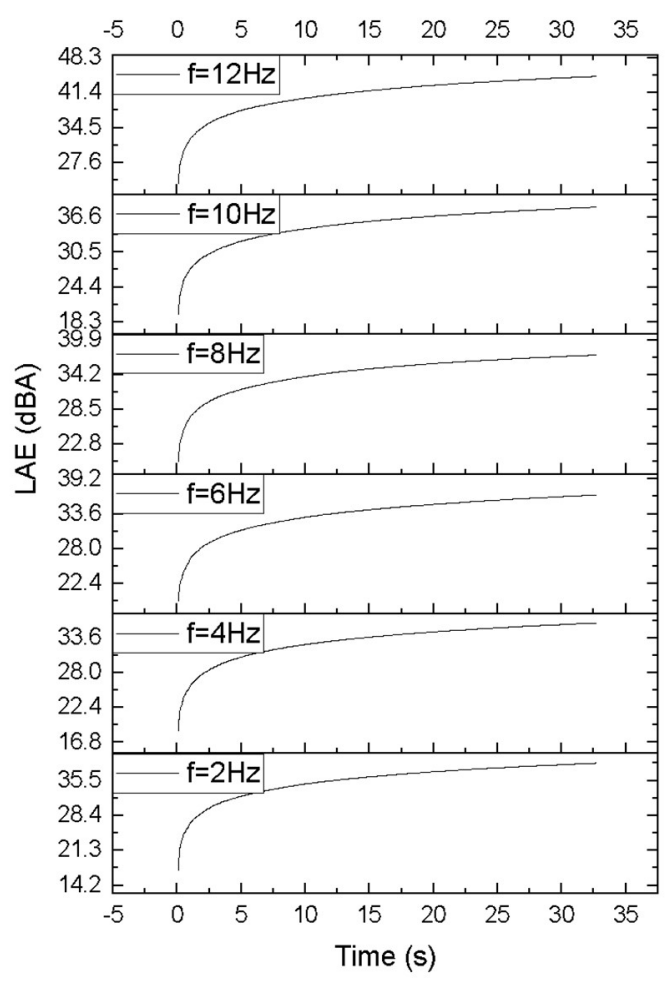

Fig. 24. Recorded noise level of PETS-flapping wing MAV with respect to time at different flapping frequencies.

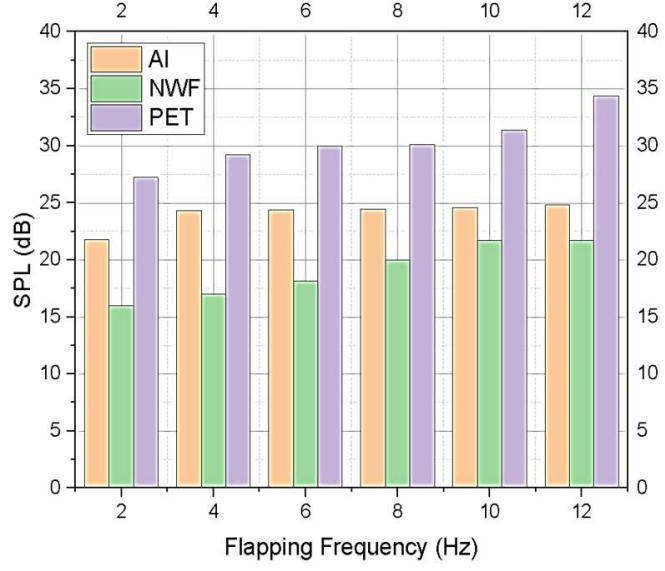

Fig. 25. Variations of Sound Pressure Level with Flapping frequency in a MAV-wing made of different materials at $\mathbf{1 8 0}^{\circ}$ location.

\section{REFERENCES}

Bae, Y. and Y. J. Moon (2008). Aerodynamic sound generation of flapping wing. The Journal of the Acoustical Society of America 124(1), 72-81.

DeLaurier, J. (1993). An aerodynamic model for flapping-wing flight. The Aeronautical Journal 97(964), 125-130.

Ifju, P., D. Jenkins, S. Ettinger, Y. Lian, W. Shyy and M. Waszak (2002). Flexible-wing-based micro air vehicles. AIAA 2002-0705.

Ifju, P., M. Waszak and L. Jenkins (2001, August). Stability and control properties of an aeroelastic fixed wing micro aerial vehicle. In proceedings of AIAA Atmospheric Flight Mechanics Conference and Exhibit. AIAA, 2001-4005, Montreal, Canada.

Lian, Y. (2003). Membrane and Adaptively-Shaped Wings for Micro Air Vehicles. Ph.D. dissertation, Mechanical and Aerospace Engineering Department, Gainesville, University of Florida.

Liebeck, R. H. (1992). Laminar separation bubbles and airfoil design at low Reynolds numbers, AIAA 1992-2735.

Lu, Z., M. Debiasi, Q. Nguyen and W. L. Chan (2014, August). An experimental investigation on the acoustic performance of a flapping wing Micro-Air-Vehicle. In proceedings of INTERNOISE 2014 - 43rd International Congress on Noise Control Engineering: Improving the World Through Noise Control. Temasek Laboratories, National University of Singapore, Singapore.

Lu, Z.. M. Debiasi, Q. Nguyen, W. L. Chanand B. Khoo (2017, December). Low-noise hyperelastic membrane wings for a flapping wing MAV. In Proceedings of INTER-NOISE and NOISE-CON Congress and Conference, Hong Kong CHINA, 1004-1995.1257-1263(7). 
S. Syam Narayanan and R. Asad Ahmed/ JAFM, Vol. 14, No. 6, pp. 1817-1826, 2021.

McIntosh, S. H., S. K. Agrawal and Z. Khan (2006). Design of a Mechanism for Biaxial Rotation of a Wing for a Hovering Vehicle. IEEE/ASME Transactions of Mechatronics 11(2), 145-153.

Miller, L. A. and C. S. Peskin (2009). Flexible clap and fling in tiny insect flight, The Journal of Experimental Biology 212(19), 3076-3090.

Narayanan, S. S., A. R. Ahmed and C. M. J. Anandh (2020). Development of artificial butterflies for the study of endangered Asiatic elephants
(Elephas Maximus Maximus) in Sri Lankan forests. International Journal of Scientific \& Technology 9(3), 4629-4632.

Narayanan S. S., A. R. Ahmed, P. V. Jijo, S. Gopinath, J. Paulraj and M. Muthukumar (2019). Experimental investigation on lift generation of flapping MAV with insect wings of various species. Aircraft Engineering and Aerospace Technology 92(2), 139-144. 